and 3) to identify the unique challenges of providing palliative input into critical care. Information was extracted from Infoflex, an electronic patient database that captures details of referral, diagnosis, patient assessment and palliative care interventions.

Results 36 patients (22 male, 14 female) median age 65.5 (range 39-94) from critical care facilities were referred to HPCT over 12 months. 21 had a diagnosis of malignancy. Reasons for admission included sepsis, multi organ failure and post-operative care/complications. The reasons for referral were symptom control, support with withdrawal of therapy and management of terminal care including support to carer. 32 out of 36 patients have died. Of these, only 11 died in a critical care unit. The HPCT was able to facilitate and achieve preferred place of death outside acute hospital for 11 patients. The biggest challenge was communication as the combination of critical illness and invasive interventions often diminished the capacity of patients to make decisions. Discontinuity of care and accepting the shift in goals from intensive treatment to palliative care was emotionally difficult for staff and family.

Conclusion Despite the challenges, it is possible and appropriate to give compassionate palliative care to dying patients and their carers in a critical care setting through good interdisciplinary team working and communication, adopting a proactive approach.

\section{P 103 \\ CHALLENGES OF PROVIDING PALLIATIVE CARE IN A CRITICAL CARE SETTING}

James Davies, Vandana Vora. Palliative Medicine, Sheffield Teaching Hospitals NHS Foundation Trust, Sheffield, United Kingdom

10.1136/bmjspcare-2014-000654.144

Background An ageing population with complex co-morbidities and rising public expectation for high quality life sustaining treatment has seen a rising demand for critical care. End of life care strategy (2008) recommends delivery of high quality service in all locations including acute hospitals.

Aim and method To retrospectively review all referrals to Hospital Palliative Care Team (HPCT) over a 12 month period from specialist and general critical care units of a large teaching hospital. The objectives were:1) to identify reasons for referral, 2 ) to assess impact of palliative care interventions on patient care 